\title{
STEM-EELS-EDS Analysis of Space Weathering Features of ANGSA Lunar Soil Samples
}

\author{
Brittany Cymes, Katherine Burgess and Rhonda Stroud

\section{U.S. Naval Research Laboratory, United States}

Introduction: Space weathering refers to processes that alter the surfaces of airless bodies exposed to the harsh environment of outer space. On the Moon, solar wind irradiation and micrometeorite bombardment are the dominant space weathering processes. These processes alter the surfaces of individual regolith grains, often producing thin $(<200 \mathrm{~nm})$ amorphous rims that can be enriched in vesicles, nanophase metallic iron inclusions $\left(\mathrm{npFe}^{0}\right)$, or a combination of these [1-3]. The roles of exposure history, mineral composition, and crystal structure in the formation of amorphous rims are not well constrained. To address these gaps in understanding, lunar soil samples were analyzed with scanning transmission electron microscopy (STEM), energy dispersive spectroscopy (EDS), and electron energy loss spectroscopy (EELS) to evaluate the systematic differences in rim characteristics as a function of exposure history and mineralogy at the individual grain scale. These efforts are part of the Apollo Next Generation Sample Analysis (ANGSA) initiative examining special samples returned during the Apollo Program that have been stored under unique conditions and remain unexamined.

Methods: Lunar soil samples representing compositionally mature, partially-shaded and exposed conditions $(72321,72501)$ and also sub-mature, permanently shaded and exposed conditions (76241, 76261) were studied. These samples were stored under ambient temperatures and will be compared to frozen analogues $(72320,76240)$ in the future. The ambient samples were prepared for STEM-EDS-EELS analysis by both ultramicrotomy, and focused ion beam (FIB) microscopy. The former afforded numerous grains per grid for trend evaluation among samples and the latter enabled examination of variation within a single grain, preservation of spatial context, and uniform thicknesses. The ultramicrotomy samples were first embedded in epoxy and sectioned to $100 \mathrm{~nm}$ using a diamond knife and then loaded onto lacey carbon $\mathrm{Cu}$ grids. The FIB samples were prepared using a FEI Helios G3 Dual Beam FIB/SEM at NRL. Sample grains were selected based on EDS analysis and by textural identification of vesicle-rich rims; spaceweathered grains often have surficial blisters [4-5]. The samples were held under vacuum at room temperature for a minimum of 48 hours to drive off adsorbed water before introduction to the UHV system of the STEM. The STEM-EELS-EDS data were acquired with the Nion UltraSTEM200-X (PRISM) at NRL. The dedicated STEM is equipped with a Gatan Enfinium ER Dual EELS and Bruker X-flash windowless SDD-EDS spectrometers. The STEM was operated at $200 \mathrm{keV}$ with a $\sim 0.1 \mathrm{~nm}$ probe diameter. The EEL spectra were acquired as spectrum images (SI). Data were analyzed using DigitalMicrograph ${ }^{\circledR}$ (Gatan) and Quantax Esprit 2.0 (Bruker) software packages.

Results and Discussion: The observed mineralogy of the samples is consistent with that reported previously: abundant calcium plagioclase and glass with accessory olivine, ilmenite, clinopyroxene, and orthopyroxene [6]. Inclusion-rich space weathered rims (i.e. those bearing $n p \mathrm{Fe}^{0}$ ) are the most common type observed and have been found on the surface of each mineral phase. The $n p F e^{0}$ found in these rims, which are generally $<150 \mathrm{~nm}$ thick, are typically within the $3-15 \mathrm{~nm}$ range with individuals $\geq 25-50 \mathrm{~nm}$ in diameter occurring less frequently. Vesicular rims are less common and are often found in association with $\mathrm{nFF}^{0}$ (Fig. 1). The observed rim geochemistry is complex. Rims of $\mathrm{Mg}$ are often found associated with $\mathrm{nFe}^{0}$ in Ca-plagioclase and ilmenite, and are interpreted to result from vapor deposition via the sputtering of adjacent $\mathrm{Mg}$-rich phases such as olivine or orthopyroxene. Olivine has likewise been 
observed to have amorphous silica rims enriched with $\mathrm{npFe}^{0}$. Several unique rim features have been observed, including $\mathrm{Ca}-\mathrm{Al}$ rims, spherical particles with unusual geochemistry, and oxidized Fe nanoparticles. Of the novel textures observed, vesicular $n p \mathrm{Fe}^{0}$ are particularly compelling due to the detection of He pockets within them via EELS-SI - both within smaller sized $n p F e^{0}$ (Fig. 2a-b) and in larger $\mathrm{nFe}^{0}$ (Fig. 2c-d). Helium is significant because it is one of the main components of solar wind and

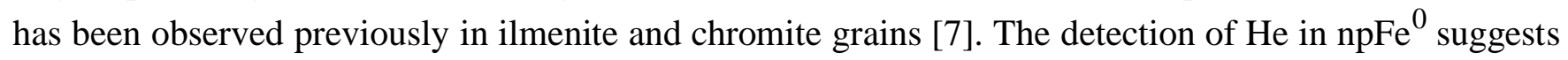
that oxide and metal particles may play a role in volatile emplacement and cycling within lunar soil. These vesicular $\mathrm{nFe}^{0}$ have been observed only in samples from full or partial exposure and thus may also represent a defining feature lacking in permanently shadowed soils.

Future Directions: The detection of He in the ambiently-stored lunar samples discussed here will provide a baseline for determining if volatiles are more effectively preserved in the frozen samples. To determine this, comparisons will be made between the density of He in vesicles [7], which will enable us to determine if cold storage slows volatile loss. We will also determine if other factors (e.g. mineral host phase) appear to affect volatile retention. By answering these questions, we will understand how the samples we currently have on Earth have changed over the past 48 years and subsequently prepare for future sample return missions and long-term storage. In addition to understanding volatile preservation, we aim to use data from these samples to ascertain the influences of solar wind irradiation and micrometeorite bombardment on the formation of space weathering features in different mineral phases and exposure contexts. Because the effects of space weathering are notably broad, affecting remote sensing measurements and potentially influencing localized albedo anomalies (i.e. lunar swirls), a systematic evaluation of samples in different exposure conditions and compositions may also provide insight into these aspects.

Acknowledgements: We acknowledge The ANGSA Science Team, and support from NASA award 80HQTR19T0057 and SSERVI award 80NSSC19M0215.

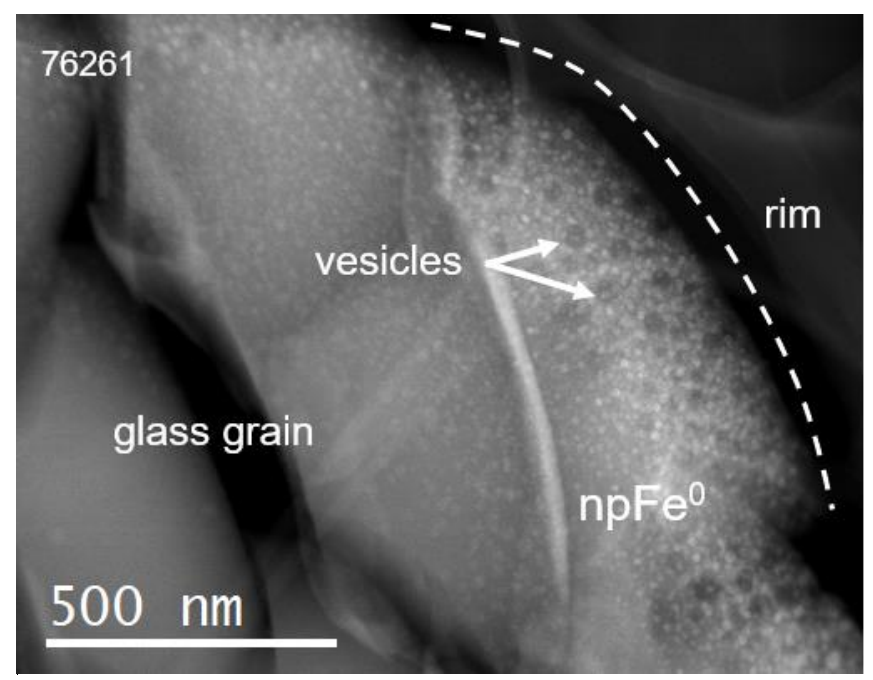

Figure 1. HAADF-STEM image of a glass grain with a space weathered rim bearing both nanophase metallic iron (npFe0) particles and vesicles. 

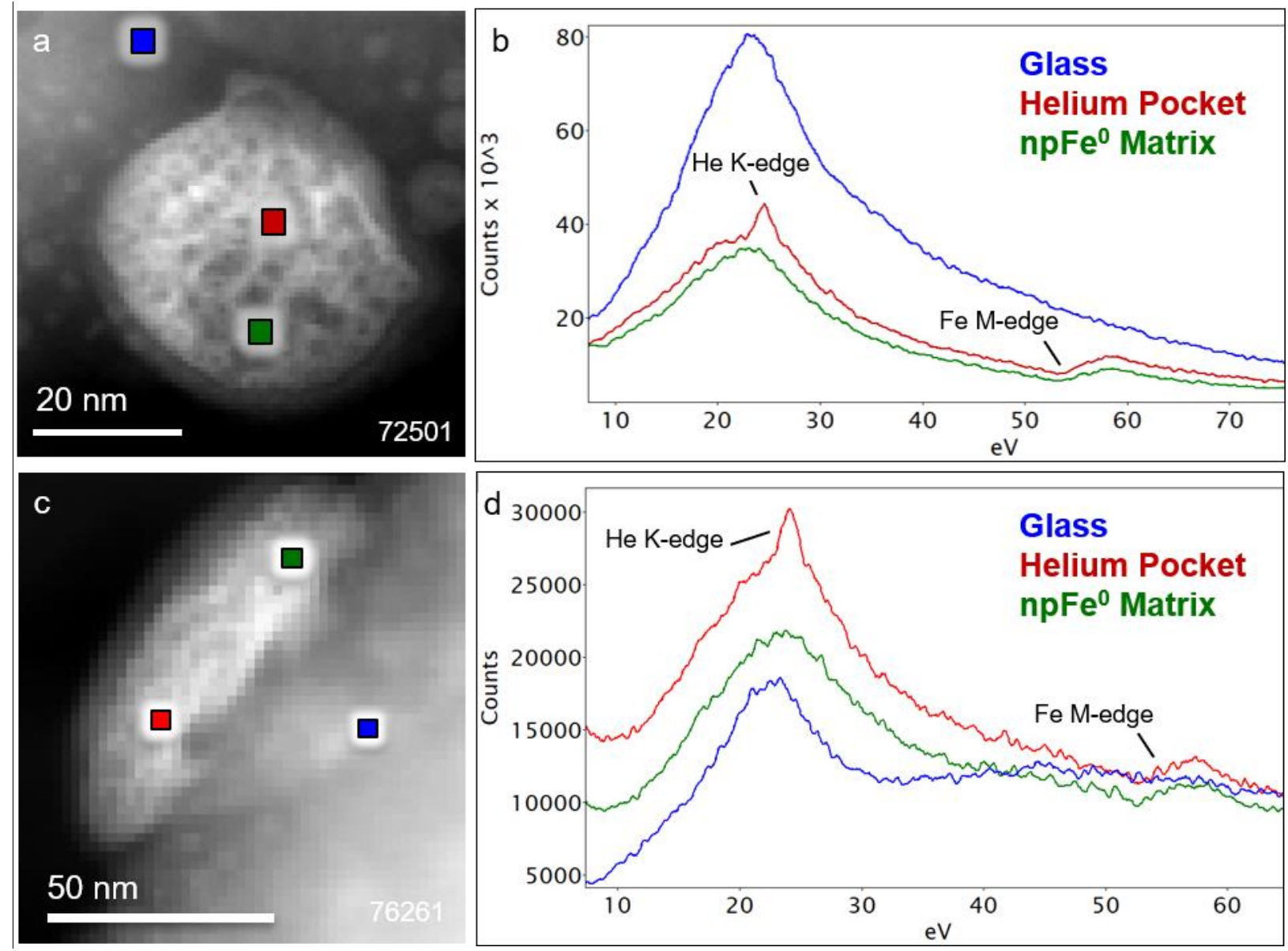

Figure 2. (a) EELS-SI of a vesicular npFe0 from sample 72501 with colored boxes indicating summed pixels from the npFe0 matrix, glass matrix, and He-pockets within the npFe0 particle. (b) Spectra from selected pixel windows in (a), (c) EELS-SI of a vesicular $\mathrm{npFe} 0$ from sample 76261 with colored boxes indicating summed pixels from the npFe0 matrix, glass matrix, and He-pockets within the npFe0 particle. (d) EEL spectra from selected pixels windows in (c).

\section{References}

[1] Pieters, C.M., Noble, S.K. (2016) J. Geophys. Res. 121, 1865-1884. [2] Hapke, B. (2001) J. Geophys. Res. 106, 10039-10073. [3] Keller, L.P., McKay, D.S. (1997) Geochim. Cosmochim. Acta 61, 2331-2341. [4] Assonov, S., Biryukov, A.Y., Kashkarov, L., Nevzorov, V., Semenova, A., Shukolyukov, Y.A. (1998) Lunar Planet. Sci. Conf. 29. [5] Matsumoto, T., Tsuchiyama, A., Miyake, A., Noguchi, T., Nakamura, M., Uesugi, K., Takeuchi, A., Suzuki, Y., Nakano, T. (2015) Icarus 257, 230-238. [6] Heiken, G., \& McKay, D. S. (1974) Lunar Planet. Sci. Conf. 5, 843-860. [7] Burgess, K.D., Stroud, R.M. (2018) Geochim. Cosmochim. Acta 224, 64-79. 